\title{
HGF and NRGI protein expression are not poor prognostic markers in surgically resected lung adenocarcinoma
}

This article was published in the following Dove Press journal:

OncoTargets and Therapy

25 May 2015

Number of times this article has been viewed

\author{
Bin $\operatorname{Pan}^{1,2}$ \\ Rui Wang ${ }^{1,2}$ \\ Yangle Huang ${ }^{1,2}$ \\ David Garfield ${ }^{3}$ \\ Jie Zhang ${ }^{1,2}$ \\ Haiquan Chen ${ }^{1,2}$ \\ 'Department of Thoracic Surgery, \\ Fudan University Shanghai Cancer \\ Center, Shanghai, People's Republic \\ of China; ${ }^{2}$ Department of Oncology, \\ Shanghai Medical College, Fudan \\ University, Shanghai, People's Republic \\ of China; ${ }^{3}$ University of Colorado \\ Comprehensive Cancer Center. \\ Aurora, CO, USA
}

Purpose: Although over-expression of hepatocyte growth factor (HGF) and neuregulin-1 (NRG1) are important mechanisms involved in acquired drug-resistance in many cancers, few reports have evaluated their clinicopathologic features and prognostic significance. The aim of our study was to investigate protein expressions of HGF and NRG1 in lung adenocarcinomas and their association with clinicopathologic parameters, oncogenic mutations, and the prognosis.

Methods: HGF and NRG1 protein tumor/stroma expressions were evaluated by immunohistochemistry (IHC) in 115 surgically resected lung adenocarcinomas and were correlated with clinicopathologic and molecular variables including tumor size, tumor node metastasis stage, differentiation, oncogenic mutations (EGFR, KRAS, HER2, BRAF) and ALK fusions, relapsefree survival, and overall survival.

Results: Positive IHC HGF tumor and stroma staining were found in 49 (42.61\%) and 12 (10.43\%) cases, respectively, while positive IHC NRG1 tumor and stroma staining were found in $56(48.70 \%)$ and eleven $(9.57 \%)$ cases, respectively. Dual positive IHC HGF and NRG1 tumor staining was $12.17 \%$. EML4-ALK fusion more significantly existed in HGF-tumor positive samples $(P=0.03)$, positive NRG1 protein stroma expression was significantly associated with male sex $(P=0.04)$, while HGF and NRG1 dual tumor-positive mainly existed in the tumor size $>3 \mathrm{~cm}$ group $(P=0.0231)$. No significant clinically prognostic difference was found between patients with HGF/NRG1-positive expression and those with HGF/NRG1-negative expression.

Conclusion: This study represents the first comprehensive analysis of HGF and NRG1 tumor and stroma expressions in patients with surgically resected lung adenocarcinomas. Our molecular data, in conjunction with clinical and pathological features, as well as their effects on survival indicated to us that patients with HGF- and NRG1-negative expression tended to have better survival, but these results probably did not warrant these markers to be indicators of poor prognosis.

Keywords: HGF, NRG1, resected lung adenocarcinoma, prognosis

\section{Introduction}

Lung cancer remains the leading cause of cancer-related death worldwide. ${ }^{1}$ Although surgical resection offers the best possibility for cure for non-small cell lung cancer (NSCLC), the great majority of surgically resected stage III and IV lung cancer patients receive additional treatment, such as chemotherapy and targeted therapy. However, the efficacy of kinase inhibitors in patients whose tumors harbor driver mutations is invariably limited by innate or acquired drug resistance. ${ }^{2,3}$ Many studies have suggested that the tumor micro-environment confers innate resistance to such therapies. In that regard, some receptor tyrosine kinase (RTK) ligands known to be
Haiquan Chen

Department of Thoracic Surgery, Fudan University Shanghai Cancer Center,

270 Dong'An Road, Shanghai 200032

People's Republic of China

Tel +86 2l 641755902500

+8621 641755901707

Email zhangjie2289@hotmail.com;

hqchenI@yahoo.com 
widely expressed in tumors such as hepatocyte growth factor (HGF) and neuregulin-1 (NRG1) may have important effects on drug response. ${ }^{4,5}$

HGF binds to its cognate c-Met receptor, whose activation results in translocation of $\beta$-catenin (CTNNB1) into the nucleus, where it acts as a transcription factor of canonical Wnt gene product, tumor MET receptor protein expression, HGF protein expression, and high MET gene copy number, all of which are indicative of poor prognosis. ${ }^{6-9}$

NRG1 encodes NRG1 (formerly the HRGs), ligands for members of the ErbB/EGFR family, which includes ErbB2/HER2. ${ }^{10}$ Overexpression of the RTK HER2/ErbB2 (ERBB2) has been linked to a poor prognosis for patients with breast cancer; thus, its activity is a central target for cancer therapy. Likewise, overexpression of HRG/NRG1, a growth factor responsible for ErbB2 activation, has been shown to be a driver of breast cancer progression. ${ }^{11}$ A recent study showed that inhibition of NRG1 signaling inhibited primary tumor growth and enhanced the magnitude and duration of the response to chemotherapy. ${ }^{12}$

Few reports have evaluated the clinicopathologic features and prognostic significance of HGF and NRG1. The aim of our work was to investigate protein expression of HGF and NRG1 in lung adenocarcinomas and their association with clinicopathologic parameters, commonly reported driver mutations, and prognosis.

\section{Materials and methods \\ Patients and samples}

Tumor specimens were obtained from patients who underwent surgical resection with curative intent at our institution from January 2008 to January 2009. We routinely performed contrast-enhanced chest computed tomography (CT) before surgery. Other routine preoperative examinations included cardiopulmonary tests, brain magnetic resonance imaging (MRI) or CT, bone scanning, and abdominal CT or ultrasonography. Positron emission tomography (PET)-CT was optional. Inclusion criteria included: 1) pathologically confirmed NSCLC; 2) sufficient tissue for comprehensive mutational analyses and immunohistochemical staining. Patients who had received neoadjuvant chemotherapy or had a history of malignant tumors were excluded. Our institutional review board approved this study, and written informed consent was obtained from all patients.

\section{Immunohistochemistry (IHC) and interpretation}

One slide section was used for each tumor specimen. The percentage of tumor cells in the sections taken for IHC analysis was at least $30 \%$. Briefly, after deparaffinization and rehydration, sections were treated with $3 \% \mathrm{H}_{2} \mathrm{O}_{2}$ to block endogenous peroxidase activity. Antigen retrieval was done by immersing slides in sodium citrate and microwaving. Non-specific Ig binding was blocked using 10\% goat serum in phosphate buffered saline. Slides were then separately incubated with anti-HGF antibody (Santa Cruz Biotechnology, inc., Dallas, TX, USA) at 1:200 and anti-NRG1 antibody (Abcam, Cambridge, MA, USA) at 1:100. After incubation with the primary antibody overnight, the sections were washed with phosphate buffered saline and incubated with secondary antibodies followed by incubation with 3,3'-diaminobenzidine (DAB). Slides were counterstained with hematoxylin. A certified pathologist (Yuan Li), who was blinded to the clinical data, assessed HGF/NRG1 cytoplasm immunestaining. For HGF and NRG1 staining, intensity $(0,1+, 2+$, $3+)$ and percentage of immunoreactive cells were recorded as followed: $3+$, strong staining intensity in $>50 \%$ cells; $2+$, moderate staining intensity in $>50 \%$ cells; $1+$, faint or weak staining intensity in $>50 \%$ cells; and 0 , no or equivocal staining in tumor cells or $<50 \%$ of cells staining at any given intensity, which were defined as HGF or NRG1-negative. Tumors with $3+, 2+$, and $1+$ intensity in $>50 \%$ tumor cells were defined as HGF or NRG1-positive. ${ }^{13}$ Mutational analyses and clinical variables RNA were extracted from frozen tumor specimens, and were reverse-transcribed into complementary DNA (cDNA). EGFR (exons 18-22), KRAS (exons 2-3), HER2 (exons 18-21), and BRAF (exons 11-15) were amplified by polymerase chain reaction using cDNA, and the amplified products were analyzed by direct dideoxynucleoside sequencing. Detailed information on detection of $A L K$ fusions was reported previously. ${ }^{14}$ Clinical variables collected included sex, age at diagnosis, smoking history, tumor differentiation, tumor size, and tumor node metastasis stage according to the seventh edition of lung cancer staging system. ${ }^{15,16}$ Disease relapse and survival were recorded on the basis of follow-up clinic or by telephone.

\section{Statistical analysis}

Statistical analyses were done with SPSS for Windows (version 16.0) and Stata (version 11.1). Correlations between different immunoreactivity and clinical variables were assessed using Pearson's chi-squared test or Fisher's exact test. Survival curves were drawn by the Kaplan-Meier method. Relapse-free survival (RFS) and overall survival (OS) of patients with positive or negative immunoreactivity were compared using the log-rank test. All tests were two-sided, and a $P$-value of less than 0.05 was considered statistically significant. 


\section{Results}

\section{Patient characteristics}

One hundred and fifteen lung adenocarcinoma samples were from 51 females and 64 males ranging in age from 34 to 80 (median, 59) years. Sixty-one patients were never-smokers. There were 70 patients with tumors that were moderately- to well-differentiated and 45 patients with poorly differentiated tumors. There were 45 patients with tumor diameters of more than $3 \mathrm{~cm}$. The number of patients in stages I-IV was 38, 24,51 , and 2, respectively. Detailed information on tumor differentiation, tumor node metastasis stage, and mutational status is listed in Table 1 . Sixty-one (53\%) patients were found to harbor EGFR kinase domain mutations (Table 1): $25(21.73 \%)$ were deletions in exon 19, 28 (24.34\%) were L858R missense changes, six (5.23\%) were exon 20 insertion, one $(0.85 \%)$ was double mutation involving exon 18 (G719S) and exon 21 (L861Q), and one (0.85\%) was double mutation involving L858R and T790M.

\section{Clinicopathologic and molecular features associated with HGF/NRGI expression}

Using the IHC scoring criteria as described above, the population was separated into $49 \mathrm{HGF}$-tumor positive patients and $66 \mathrm{HGF}$-tumor negative patients, $12 \mathrm{HGF}$-stroma positive patients and $103 \mathrm{HGF}$-stroma negative patients. There were 56 NRG1-tumor positive patients and 59 NRG1-tumor negative patients, eleven NRG1-stroma positive patients and 104 NRG1-stroma negative patients. Examples of IHC scoring criteria are provided in Figure 1. As shown in Table 2, EML4-ALK fusions more significantly existed in HGF-tumor positive tumors $(P=0.03)$. Positive NRG1 stroma expression
Table I Detailed clinicopathologic characteristics of II5 lung adenocarcinomas

\begin{tabular}{|c|c|}
\hline Variable & n (\%) \\
\hline Total patients & 115 (100.0\%) \\
\hline \multicolumn{2}{|l|}{ Age } \\
\hline$\leq 60$ & $64(55.6 \%)$ \\
\hline$>60$ & $5 \mathrm{I}(44.4 \%)$ \\
\hline \multicolumn{2}{|l|}{ Sex } \\
\hline Male & $64(55.6 \%)$ \\
\hline Female & $5 \mathrm{I}(44.4 \%)$ \\
\hline \multicolumn{2}{|l|}{ Smoking } \\
\hline Never-smoker & $6 \mathrm{I}(53.0 \%)$ \\
\hline Smoker & $54(47.0 \%)$ \\
\hline \multicolumn{2}{|l|}{ Differentiation } \\
\hline Moderate to well & 70 (60.9\%) \\
\hline Poor & 45 (39.1\%) \\
\hline \multicolumn{2}{|l|}{ Tumor size $(\mathrm{cm})$} \\
\hline$\leq 3$ & 70 (60.9\%) \\
\hline$>3$ & 45 (39.1\%) \\
\hline \multicolumn{2}{|l|}{ TNM stage } \\
\hline I-II & $62(53.9 \%)$ \\
\hline III-IV & $53(46.1 \%)$ \\
\hline \multicolumn{2}{|c|}{ Mutation status of EGFR } \\
\hline Wild & $54(47.0 \%)$ \\
\hline Mutation & $6 \mathrm{I}(53.0 \%)$ \\
\hline \multicolumn{2}{|c|}{ Mutation status of KRAS } \\
\hline Wild & $109(94.8 \%)$ \\
\hline Mutation & $6(5.2 \%)$ \\
\hline \multicolumn{2}{|c|}{ Mutation status of HER2 } \\
\hline Wild & 115 (100.0\%) \\
\hline Mutation & 0 \\
\hline \multicolumn{2}{|c|}{ Mutation status of BRAF } \\
\hline Wild & $115(100.0 \%)$ \\
\hline Mutation & 0 \\
\hline \multicolumn{2}{|c|}{ Fusion status of EML4-ALK } \\
\hline Wild & $106(92.1 \%)$ \\
\hline Fusion & $9(7.9 \%)$ \\
\hline
\end{tabular}

Abbreviation: TNM, tumor node metastasis.
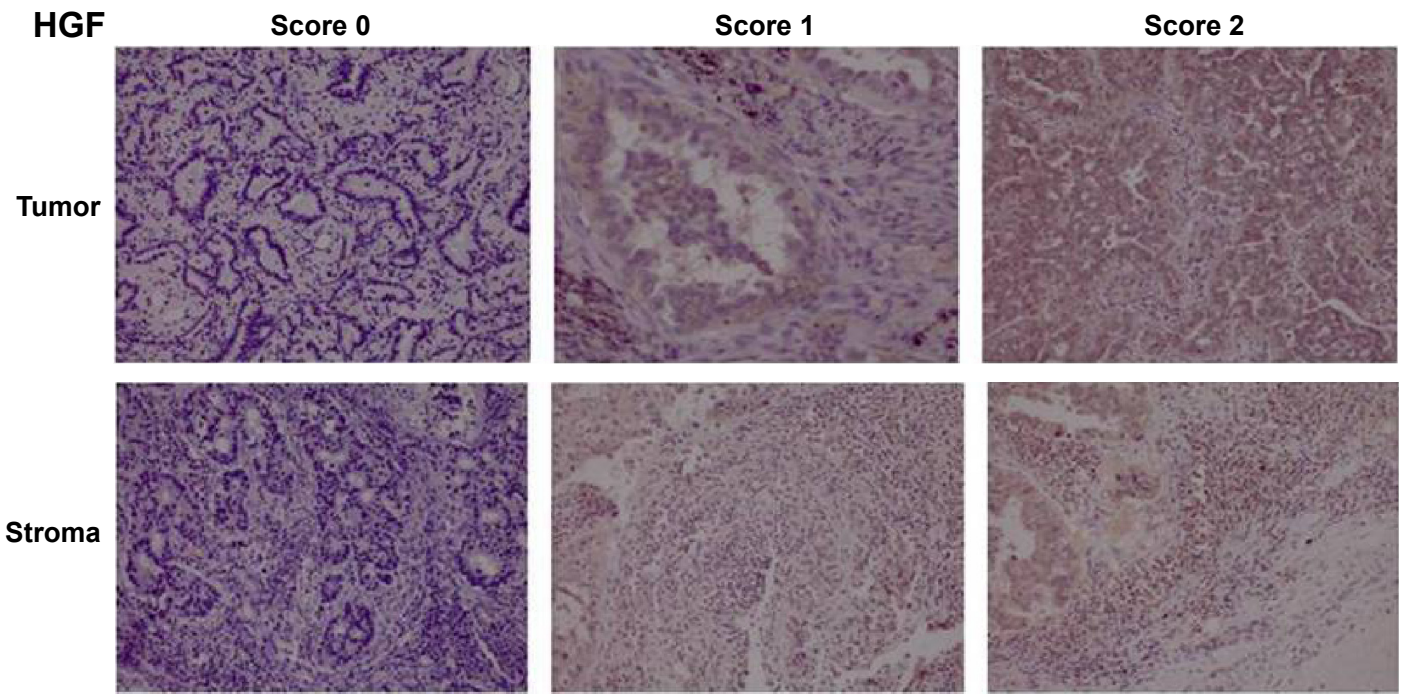

Figure I (Continued) 

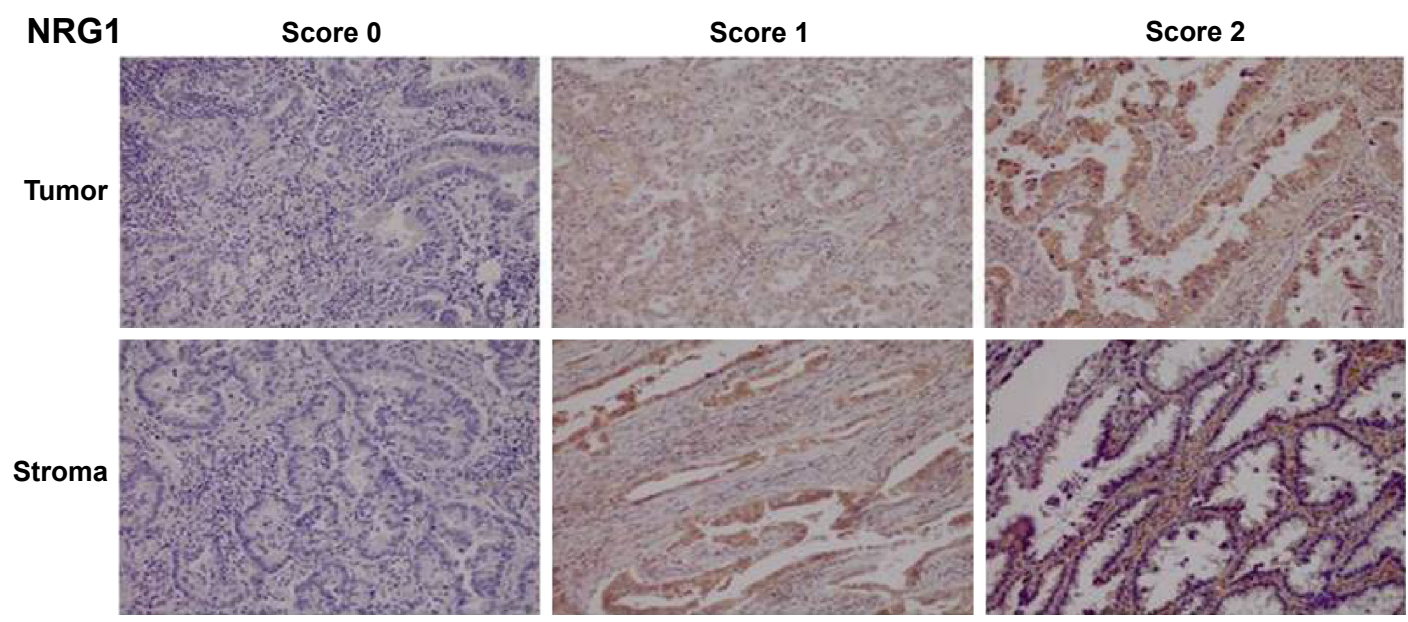

Figure I Immunohistochemical staining with HGF and NRGI in lung adenocarcinoma.

Abbreviations: HGF, hepatocyte growth factor; NRGI, neuregulin-I.

Table 2 Association between clinicopathologic characteristics and expressions of HGF or NRGI

\begin{tabular}{|c|c|c|c|c|c|c|c|c|c|c|c|c|}
\hline \multirow[t]{2}{*}{ Variable } & \multicolumn{2}{|l|}{ HGF } & \multirow[t]{2}{*}{$P$} & \multicolumn{2}{|l|}{ HGF } & \multirow[t]{2}{*}{$P$} & \multicolumn{2}{|l|}{ NRG I } & \multirow[t]{2}{*}{$P$} & \multicolumn{2}{|c|}{ NRG I } & \multirow[t]{2}{*}{$P$} \\
\hline & $\mathbf{T}(+)$ & $\mathbf{T}(-)$ & & $\mathbf{S}(+)$ & S (-) & & $\mathbf{T}(+)$ & $\mathbf{T}(-)$ & & $\mathbf{S}(+)$ & S (-) & \\
\hline Total patients & 49 & 66 & & 12 & 103 & & 56 & 59 & & 11 & 104 & \\
\hline Age & & & 0.16 & & & 0.30 & & & 0.42 & & & 0.25 \\
\hline$\leq 60$ & 31 & 33 & & 5 & 59 & & 29 & 35 & & 6 & 58 & \\
\hline$>60$ & 18 & 33 & & 7 & 44 & & 27 & 24 & & 5 & 46 & \\
\hline Sex & & & 0.75 & & & 0.38 & & & 0.65 & & & 0.04 \\
\hline Male & 26 & 37 & & 8 & 55 & & 32 & 31 & & 9 & 54 & \\
\hline Female & 23 & 29 & & 4 & 48 & & 24 & 28 & & 2 & 50 & \\
\hline Smoking & & & 0.99 & & & 0.40 & & & 0.42 & & & 0.07 \\
\hline Never-smoker & 26 & 35 & & 5 & 56 & & 29 & 32 & & 3 & 58 & \\
\hline Smoker & 23 & 31 & & 7 & 47 & & 27 & 27 & & 8 & 46 & \\
\hline Differentiation & & & 0.95 & & & 0.76 & & & 0.46 & & & 0.25 \\
\hline Moderate to well & 30 & 40 & & 8 & 62 & & 36 & 34 & & 7 & 63 & \\
\hline Poor & 19 & 26 & & 4 & 41 & & 20 & 25 & & 4 & 41 & \\
\hline Tumor size $(\mathrm{cm})$ & & & 0.14 & & & 024 & & & 0.68 & & & 0.11 \\
\hline$\leq 3$ & 26 & 44 & & 7 & 63 & & 33 & 37 & & 4 & 66 & \\
\hline$>3$ & 23 & 22 & & 5 & 40 & & 23 & 22 & & 7 & 38 & \\
\hline TNM stage & & & 0.59 & & & 0.23 & & & 0.07 & & & 0.49 \\
\hline I-II & 25 & 37 & & 7 & 55 & & 35 & 27 & & 7 & 55 & \\
\hline III-IV & 24 & 29 & & 5 & 48 & & 21 & 32 & & 4 & 49 & \\
\hline Mutation status of EGFR & & & 0.13 & & & 0.82 & & & 0.31 & & & 0.64 \\
\hline Wild & 27 & 27 & & 6 & 48 & & 29 & 25 & & 5 & 49 & \\
\hline Mutation & 22 & 39 & & 6 & 55 & & 27 & 34 & & 6 & 55 & \\
\hline Mutation status of KRAS & & & 0.16 & & & 0.49 & & & 0.68 & & & 0.56 \\
\hline Wild & 48 & 61 & & 11 & 98 & & 54 & 55 & & 11 & 98 & \\
\hline Mutation & 1 & 5 & & 1 & 5 & & 2 & 4 & & 0 & 6 & \\
\hline Mutation status of HER2 & & & 1.00 & & & 1.00 & & & 1.00 & & & 1.00 \\
\hline Wild & 49 & 66 & & 12 & 103 & & 56 & 59 & & 11 & 104 & \\
\hline Mutation & 0 & 0 & & 0 & 0 & & 0 & 0 & & 0 & 0 & \\
\hline Mutation status of BRAF & & & 1.00 & & & 1.00 & & & 1.00 & & & 1.00 \\
\hline Wild & 49 & 66 & & 12 & 103 & & 56 & 59 & & 11 & 104 & \\
\hline Mutation & 0 & 0 & & 0 & 0 & & 0 & 0 & & 0 & 0 & \\
\hline Fusion status of EML4-ALK & & & 0.03 & & & & & & 0.31 & & & 1.00 \\
\hline Wild & 42 & 64 & & 12 & 94 & 1.00 & 50 & 56 & & 11 & 95 & \\
\hline Fusion & 7 & 2 & & 0 & 9 & & 6 & 3 & & 0 & 9 & \\
\hline
\end{tabular}

Abbreviations: T, tumor; S, stroma; (+), positive; (-), negative; TNM, tumor node metastasis; HGF, hepatocyte growth factor; NRGI, neuregulin-I. 
was significantly associated with male sex $(P=0.04)$. HGF or NRG1 expression did not differ significantly in other clinicopathologic characteristics variables. As shown in Table 3, HGF and NRG1 dual tumor-positive mainly existed in the tumor size $>3 \mathrm{~cm}$ group, while dual tumor-negative mainly existed in the tumor size $\leq 3 \mathrm{~cm}$ group $(P=0.02)$.

\section{Survival analysis}

By univariate analysis, patients with positive HGF expression tended to have a worse RFS and OS compared with the negative HGF expression group, although this was without statistical significance (RFS: $P=0.0895$; OS: $P=0.0951$ ). Also, there were no significant differences of RFS and OS between patients with positive NRG1 expression and negative NRG1 expression (Figure 2). HGF and NRG1 dual tumor-positive mainly existed in the tumor size $>3 \mathrm{~cm}$ patients (Table 3). There were no significant differences of RFS and OS between patients of HGF and NRG1 dual tumor-positive and the dual tumor-negative groups (Figure 3).

\section{Discussion}

NSCLC is a heterogeneous disease, and disease outcome varies even in patients with apparently identical clinicopathologic features. IHC analysis is able to add prognostic value to the current staging system through identifying markers of tumor aggressiveness.

Many studies have shown that some RTK ligands known to be widely expressed in tumors, for example, HGF and NRG1, may have important effects on drug responses, ${ }^{4,5}$ and high circulating HGF levels have been associated with epithelial to mesenchymal transition and poor outcome in small cell lung cancer patients. ${ }^{17}$ NRG1 signaling in these models can be mediated by either the HER3 or HER4 receptor,
Table 3 Association between clinicopathologic characteristics and tumor expressions of HGF and NRGI

\begin{tabular}{|c|c|c|c|}
\hline \multirow[t]{2}{*}{ Variable } & \multicolumn{2}{|c|}{ HGF and NRG I } & \multirow[t]{2}{*}{$P$} \\
\hline & $\mathbf{T}(+)$ & $\mathbf{T}(-)$ & \\
\hline Total patients & 14 & 17 & \\
\hline Age & & & 0.51 \\
\hline$\leq 60$ & 9 & 12 & \\
\hline$>60$ & 5 & 5 & \\
\hline Sex & & & 0.28 \\
\hline Male & 13 & 16 & \\
\hline Female & I & 1 & \\
\hline Smoking & & & 1.00 \\
\hline Never-smoker & 0 & 0 & \\
\hline Smoker & 14 & 17 & \\
\hline Differentiation & & & 0.18 \\
\hline Moderate to well & 9 & 8 & \\
\hline Poor & 5 & I & \\
\hline Tumor size $(\mathrm{cm})$ & & & 0.02 \\
\hline$\leq 3$ & 5 & 13 & \\
\hline$>3$ & 9 & 4 & \\
\hline TNM stage & & & 0.12 \\
\hline I-II & 10 & 8 & \\
\hline III-IV & 4 & 9 & \\
\hline Mutation status of EGFR & & & 0.21 \\
\hline Wild & 7 & II & \\
\hline Mutation & 7 & 6 & \\
\hline Mutation status of KRAS & & & 1.00 \\
\hline Wild & 14 & 15 & \\
\hline Mutation & 0 & 2 & \\
\hline Mutation status of HER2 & & & 1.00 \\
\hline Wild & 14 & 17 & \\
\hline Mutation & 0 & 0 & \\
\hline Mutation status of BRAF & & & 1.00 \\
\hline Wild & 14 & 17 & \\
\hline Mutation & 0 & 0 & \\
\hline Fusion status of EML4-ALK & & & 1.00 \\
\hline Wild & 13 & 17 & \\
\hline Fusion & I & 0 & \\
\hline
\end{tabular}

Abbreviations: T, tumor; (+), positive; (-), negative; TNM, tumor node metastasis; HGF, hepatocyte growth factor; NRGI, neuregulin-I.
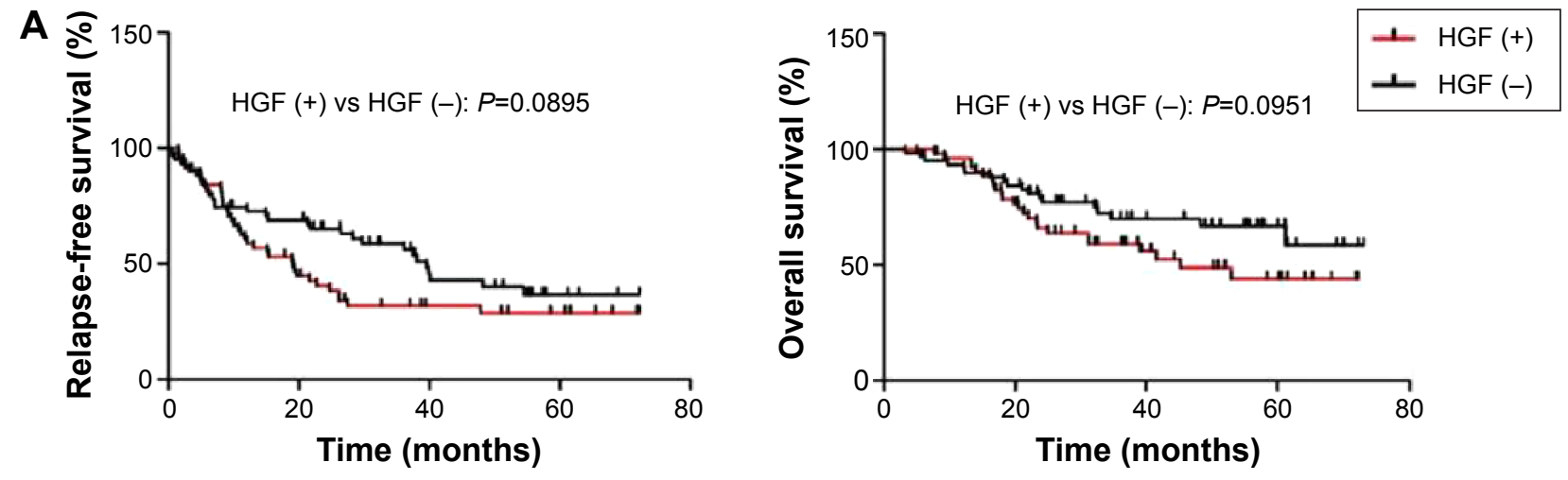

Figure 2 (Continued) 

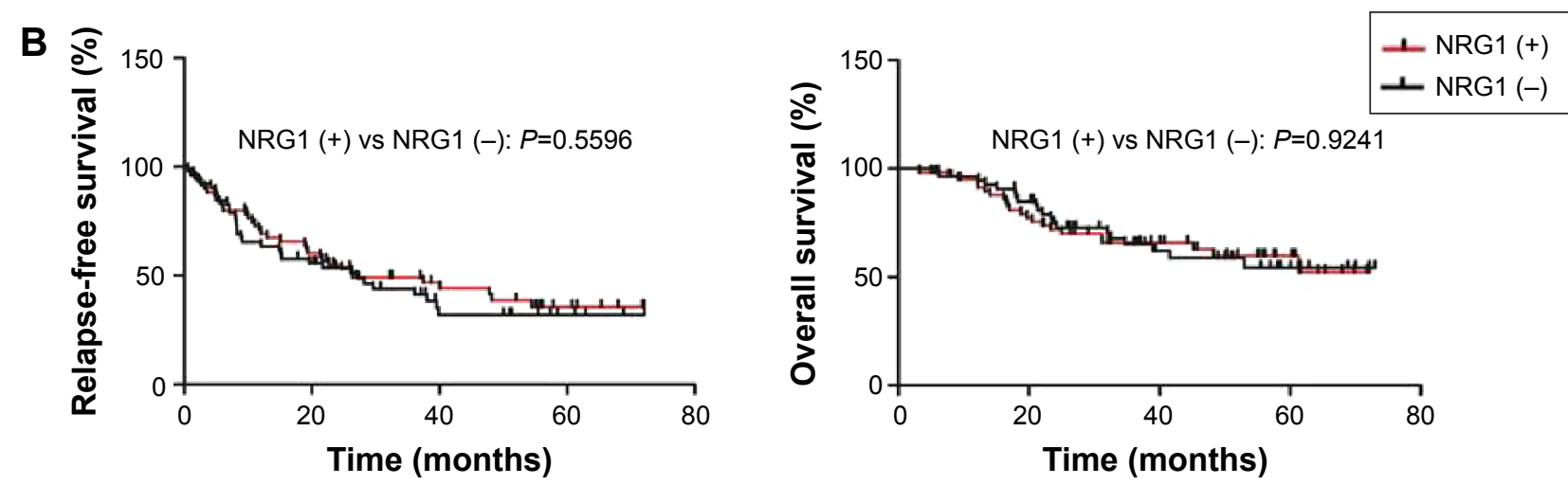

Figure 2 Relapse-free survival and overall survival in patients with lung adenocarcinoma based on HGF (A) or NRGI (B) expression. Abbreviations: (+), positive; (-), negative; HGF, hepatocyte growth factor; NRGI, neuregulin-I.

resulting in differential activation of downstream effectors. Inhibition of NRG1 signaling inhibited primary tumor growth and enhanced the magnitude and duration of the response to chemotherapy in NSCLC models. ${ }^{12}$

We have investigated the correlation between HGF/ NRG1 expression and patient characteristics, tumor pathology as well as well-identified driver mutations in lung adenocarcinomas. EML4-ALK fusion was more frequently found in HGF tumor-positive patients. This suggests that the HGF-positive tumors may be biologically more responsive to ALK inhibitor treatment. Positive NRG1 stroma expression was significantly more frequently found in males, which was not reported in other studies. Although NRG1 stroma expression in males and females are different, the survival of different sex and NRG1 different expression was not significantly correlated. HGF and NRG1 dual tumor-positive mainly existed in tumor size $>3 \mathrm{~cm}$ patients. This may partly explain why HGF and NRG1 dual tumor-positive patients tended to have worse RFS and OS compared with the dual tumor-negative group, although it was without statistical significance. Collectively, our study suggests that patients with HGF- and NRG1-negative expression tended to have better survival, but positive expression of either was not necessarily a marker of poor prognosis.

We acknowledge that there are several limitations of this study. First, as IHC was used as the primary method to assess HGF/NRG1 status, there is the issue of the interobserver variability. Second, this study was retrospective, and the sample size may not have been sufficiently large. Therefore, a prospective study is warranted in order to validate the application of HGF/NRG1 in the identification of high-risk lung adenocarcinoma patients who might benefit from adjuvant treatment.

In conclusion, through IHC analysis of 115 surgically resected lung adenocarcinomas, we found that patients with HGF- and NRG1-negative expressions tended to have better OS, but this probably did not warrant these as markers of poor prognosis. Prospective studies are warranted to validate their prognostic value in conjunction with the current lung cancer staging system.

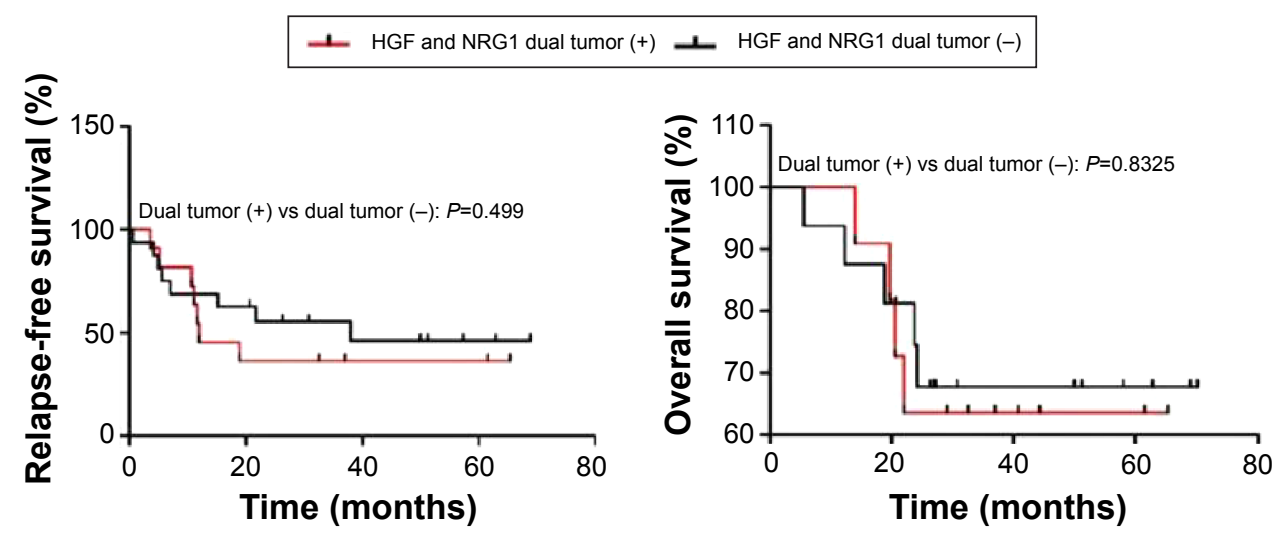

Figure 3 Relapse-free survival and overall survival in patients with lung adenocarcinoma based on HGF and NRGI tumor expression. Abbreviations: $(+)$ positive, (-) negative; HGF, hepatocyte growth factor; NRGI, neuregulin- I; vs, versus. 


\section{Acknowledgments}

This work is supported, in part, by Shanghai Science and Technology Commission Foundation Key Project, Longhua Medicial Project, Young Investigator grant from Shanghai Municipal Health Bureau, Zhuo Shi grant from Fudan University, Ministry of Education Returned Scientific Research Foundation, and Trans-Century Training Programme Foundation for the Talents by the State Education Commission (to JZ).

\section{Disclosure}

The authors report no conflicts of interest in this work.

\section{References}

1. Jemal A, Bray F, Center MM, et al. Global cancer statistics. CA Cancer J Clin. 2011;61(2):69-90

2. Sequist LV, Waltman BA, Dias-Santagata D, et al. Genotypic and histological evolution of lung cancers acquiring resistance to EGFR inhibitors. Sci Transl Med. 2011;3(75):75ra26.

3. Garrett JT, Arteaga CL. Resistance to HER2-directed antibodies and tyrosine kinase inhibitors: mechanisms and clinical implications. Cancer Biol Ther. 2011;11(9):793-800.

4. Straussman R, Morikawa T, Shee K, et al. Tumor microenvironment elicits innate resistance to RAF inhibitors through HGF secretion. Nature. 2012;487(7408):500-504

5. Wilson TR, Fridlyand J, Yan Y, et al. Widespread potential for growthfactor-driven resistance to anticancer kinase inhibitors. Nature. 2012; 487(7408):505-509.

6. Masuya D, Huang C, Liu D, et al. The tumour-stromal interaction between intratumoral c-Met and stromal hepatocyte growth factor associated with tumour growth and prognosis in non-small cell lung cancer patients. Br J Cancer. 2004;90(8):1555-1562.

7. Cappuzzo F, Marchetti A, Skokan M, et al. Increased MET gene copy number negatively affects survival of surgically resected non-small cell lung cancer patients. J Clin Oncol. 2009;27(10):1667-1674.
8. Beau-Faller M, Ruppert A, Voegeli A, et al. MET gene copy number in non-small cell lung cancer: molecular analysis in a targeted tyrosine kinase inhibitor naive cohort. $J$ Thorac Oncol. 2008;3(4):331-339.

9. Siegfried JM, Weissfeld LA, Luketich JD, et al. The clinical significance of hepatocyte growth factor for non-small cell lung cancer. Ann Thorac Surg. 1998;66(6):1915-1918.

10. Huang HE, Chin SF, Ginestier C, et al. A recurrent chromosome breakpoint in breast cancer at the NRG1/neuregulin1/heregulin gene. Cancer Res. 2004;64(19):6840-6844.

11. Lin MC, Rojas KS, Cerione RA, Wilson KF. Identification of mTORC2 as a necessary component of HRG/ErbB2-dependent cellular transformation. Mol Cancer Res. 2014;12(6):940-952.

12. Hegde GV, de la Cruz CC, Chiu C, et al. Blocking NRG1 and other ligand-mediated Her4 signaling enhances the magnitude and duration of the chemotherapeutic response of non-small cell lung. Sci Transl Med. 2013;5(171):171ra18.

13. Hartmut Koeppen, Wei Yu, Jiping Zha, et al. Biomarker Analyses from a Placebo-Controlled Phase II Study Evaluating Erlotinib \pm Onartuzumab in Advanced Non-Small Cell Lung Cancer: MET Expression Levels Are Predictive of Patient Benefit. Clin Cancer Res. 2014;20(17): 4488-4498.

14. Wang R, Pan Y, Li C, et al. The use of quantitative real-time reverse transcriptase PCR for 50 and 30 portions of ALK transcripts to detect ALK rearrangements in lung cancers. Clin Cancer Res. 2012;18(17): 4725-4732.

15. Detterbeck FC, Boffa DJ, Tanoue LT. The new lung cancer staging system. Chest. 2009;136(1):260-271.

16. Travis WD, Brambilla E, Noguchi M, et al. International Association for the Study of Lung Cancer/American Thoracic Society/European Respiratory Society International multidisciplinary classification of lung adenocarcinoma. $J$ Thorac Oncol. 2011;6(2):244-285.

17. Israel Cañadas, Álvaro Taus, Iria González, et al. High circulating hepatocyte growth factor levels associate with epithelial to mesenchymal transition and poor outcome in small cell lung cancer patients. Oncotarget. 2014;5(14):5246-5256.
OncoTargets and Therapy

\section{Publish your work in this journal}

OncoTargets and Therapy is an international, peer-reviewed, open access journal focusing on the pathological basis of all cancers, potential targets for therapy and treatment protocols employed to improve the management of cancer patients. The journal also focuses on the impact of management programs and new therapeutic agents and protocols on

\section{Dovepress}

patient perspectives such as quality of life, adherence and satisfaction. The manuscript management system is completely online and includes a very quick and fair peer-review system, which is all easy to use. Visit http://www.dovepress.com/testimonials.php to read real quotes from published authors. 